\title{
Effects of Imagery Tennis Training on Cerebral Activity
}

\author{
Seokwon Jung ${ }^{1}$, Min-sun Choi ${ }^{1}$, Min-uk Kim ${ }^{1}$, Hye-jin $\mathrm{An}^{1}$, Min-gyeong Shin ${ }^{1}$ and Oh-Young Kwon ${ }^{1,2,3}$ \\ ${ }^{1}$ Department of Neurology, Gyeongsang National University Hospital, Jinju 660-702, Korea \\ ${ }^{2}$ Department of Neurology, Gyeongsang National University School of Medicine, Jinju 660-701, Korea \\ ${ }^{3}$ Gyeongsang Institute of Health Science, Gyeongsang National University, Jinju 660-702, Korea
}

\begin{abstract}
The previous studies showed that the visual imagery activated the occipital and posterior inferior temporal area of the brain, and the damage to the occipital cortex impaired the visual mental imagery. We studied current-source distribution of electroencephalography (EEG) to observe neuronal activity during imagery tennis playing. Eleven healthy volunteers were enrolled. All volunteers were right-handed males and novices for tennis playing. The mean age of them was 24.9 years. The EEGs were recorded on the scalp electrodes located according to the International $10 \sim 20$ System. The number of electrodes was 25 channels including subtemporal electrodes. The EEG recording session was 13 min including 5 segments: resting-I, scenery-slide show, resting-II, watching tennis-game video, and imagery-tennis playing. The recoding durations were 3, 2, 3, 2, and 3 min respectively. Five 'artifact free 3 -sec segments' were selected in each segment of 'imagery-tennis playing' and 'resting-Il'. We did the frequency domain analysis with the EEG segments using a distributed model of current-source analysis. The statistical-nonparametric maps (SnPMs) were obtained between the segments of 'imagery-tennis playing' and the segments of 'resting-II' $(p<0.01)$. The significant change of current-source density was observed only in alpha-2 frequency band $(10 \sim 12 \mathrm{~Hz})$. The current-sourcedensity was increased in the hippocampus, parahippocampus, and occipital fusiform gyrus in the right cerebral hemisphere $(p<0.01)$. Imaginary-tennis playing may activate the hippocampal-occipital alpha networks of nondominant hemisphere.
\end{abstract}

Keywords: Mental processes, Electroencephalography, Computer-assisted image processing

This is an Open Access article distributed under the terms of the Creative Commons Attribution Non-Commercial License (http://creativecommons.org/licenses/by-nc/3.0) which permits unrestricted non-commercial use, distribution, and reproduction in any medium, provided the original work is properly cited.

Copyright ( 2015 The Korean Society for Clinical Laboratory Science. All rights reserved.
Corresponding author: Oh-Young Kwon Department of Neurology, Gyeongsang National University School of Medicine, Jinju 660-751, Korea

Tel: 82-55-750-8288

E-mail: mnkwon21@hanmail.net

Received: February 27, 2015

Revised: March 17, 2015

Accepted: March 18, 2015

\section{서 론}

육체를 사용하지 않고 운동 행위를 마음 속에 떠올려서 특정한 운동을 훈련하는 것이 심상훈련이다. 스포츠훈련은 육체훈련이 주 된 방법이다. 심상훈련은 육체적 과정을 빼고 지식과 무의식과정 만을 가지고 훈련하여 육체훈련과 같은 효과를 얻으려고 하는 것이 다. 심상훈련을 스포츠훈련에 적용할 때에는 육체적 훈련과 병행하 는 것이 일반적이다(Jackson 등, 2001). 테니스의 서비스훈련을 하 고 나서 운동능력을 분석한 연구가 있다(Atienza 등, 1998). 이 연 구에서는 9 12세 사이 연령의 테니스 선수들에게 육체훈련만 시 키거나 심상훈련을 병행하였다. 심상훈련은 비디오를 이용하기도 하고 상상만으로 하기도 하였다. 24 주간의 훈련을 받은 뒤에, 육체 훈련만 받은 경우보다 심상훈련을 병행한 경우에서 서비스 능력이 의미 있게 향상되었다. 심상훈련을 뇌졸중 환자들의 재활에 이용할
수도 있다. 뇌졸중 환자들이 보행훈련을 할 때 심상훈련을 병행하 면 몸의 중심을 잡는 기능과 보행능력이 현저히 증진된다(Cho 등, 2013).

심상훈련은 시각상상을 이용한다. 시각상상이 뇌의 후두엽과 아 래측두옆 부위를 활성화시킨다는 것은 사건유발전위(event related potentials) (Farah 등, 1988), 단일양자방출전산화단층촬영(single photon emission computed tomography) (Goldenberg 등, 1989)과 양자방출단층촬영(photon emission tomography) (Kosslyn, 1993) 같은 기능영상들을 이용한 연구에서 밝혀졌다. 동 물실험에서는 쥐의 한쪽 후두엽을 제거하면 심상 속의 눈의 시야각 (visual angle)이 좁아져서 시각적 심상훈련에 방해를 받는다는 연 구 결과도 있다(Farah 등, 1992). 심상비행훈련을 통해 뇌파활성의 변화를 관찰한 연구에서는 심상비행훈련을 할 때 알파-2 (10 12 $\mathrm{HZ}$ )영역 파워스펙트럼이 비동기화된 결과도 있다. 초보 비행사의 
경우는 심상훈련을 하기 전에 비행기 조종에 대한 정보를 전달 받 고 대기하는 동안에도 고주파알파 비동기화가 발생하였지만 숙련 된 전투비행사에서는 심상훈련을 하는 동안에만 비동기화가 관찰 되었다(Tokumaru, 2003).

본 연구는 심상훈련의 효과를 뇌파의 전류원분석으로 연구하였 다. 두피에 기록된 전위를 수학적 방법을 통해 분석하여 전류원의 위치를 찾는 과정이 뇌파의 전류원분석이다. 이는 뇌의 전기활성을 시간적-공간적으로 파악할 수 있는 일종의 전기기능영상이다. 전 류원을 찾는 방법에는 뚜렷하게 구별이 되는 쌍극자(dipole)를 찾 아내는 분리전류원모델(discrete current source model)과 전류 원의 분포만을 찾아내는 분산전류원모델(distributed current source model)이 있다. LORETA는 분산전류원모델에 해당하는 전류원분석방법이다(Pascual-Marqui, 1994) 전류원분석을 통해 뇌안에서 전기적 활성이 심상훈련에 의해 어떠한 변화가 있는지 관 찰한 연구는 찾아보기 힘들다. 본 연구는 테니스심상훈련이 뇌 속 에 발생시키는 변화를 전류원분석을 통해 관찰하고자 하였다.

\section{재료 및 방법}

\section{1. 연구대상}

처음에는 14 명의 건강한 성인 남성이며 테니스 초보자가 대상 이 되었다. 오른손잡이 여부는 Chapman-Chapman손잡이척도 (Chapman-Chapman handedness scale)를 기준으로 정하였다 (Chapman와Chapman, 1987). 14명중에 3명이 제외되었는데, 1 명은 기록한 뇌파에 잡파가 많아 분석이 어려웠고, 2 명은 왼손잡이 로 판단되어 대상에서 제외되었다. 오른손 잡이만 선택한 이유는 우성반구의 차이가 결과에 영향을 미칠 가능성을 제거하기 위한 것 이었다. 대상으로 남은 11 명의 뇌파를 분석하였다. 이들의 평균연 령은 $24.9 \pm 0.9$ 세(평균 \pm 표준편차)였다. 문진을 통해 내과질환, 정신과질환, 약물중독 등의 과거력이 없다는 것을 확인하였고 실험 과정에 대해 설명하고 동의를 얻었다.

\section{2. 뇌파 기록}

뇌파 기록은 32채널 디지털뇌파기(Grass Telefactor, Comet $R$, $\mathrm{USA}$ )를 이용하였다. 기본 전극(Fp1/2, F7/8, T7/8, P7/8, F3/4, $\mathrm{C} 3 / 4, \mathrm{P} 3 / 4, \mathrm{O} 1 / 2, \mathrm{Fz}, \mathrm{Cz}, \mathrm{Pz})$ 에 아래관자전극(F9/10, T9/10, $\mathrm{P} 9 / 10)$ 을 포함하여 25개의 전극을 실험 대상자 두피에 부착하였 다. 전극의 위치는 국제 10 20계(International 10 20 system) 을 기준으로 하였다. 디지털뇌파 자료의 표본율은 $400 \mathrm{~Hz}$ 였다. 뇌 파는 총 13 분을 기록하였고 5 개의 실험 구간으로 나누었다. 각 구 간은 휴식-I 3분, 풍경슬라이드쇼보기 2분, 휴식-II 3분, 테니스비
디오클립보기 2 분, 그리고 테니스심상훈련 3 분의 순서로 배열하였 다. 테니스비디오클립은 서브를 넣은 장면부터 시작해서 단순한 스 트로크의 반복을 편집하여 복잡한 기술에 대한 상상은 피하도록 하 였다. 테니스심상훈련 과정 3 분 동안에는 실험대상인 사람은 눈을 감고 심상훈련을 하게하였다. 이 과정 동안 비디오클립을 본 기억 을 가지고 자신이 스트로크를 하고 있는 것을 상상하게 하였다.

\section{3. 뇌파절편}

테니스심상훈련 3 분과 휴식-II 2 분 동안 기록한 뇌파에서 잡파 가 없는 3 초 구간 5 군데를 선택하였다. 한 명의 실험 참가자당 10 개 의 3 초 구간이 선택되어 전체적으로 110 개의 뇌파절편을 모았다. 테니스심상훈련의 절편과 휴식-II 절편이 각각 55 개씩이었다. 뇌 파절편의 선택은 BESA $($ (brain electrical source analysis) 소프 트웨어를 이용하였다.

\section{4. 뇌파 전류원분석}

뇌파 전류원분석은 주파수영역으로 분석하였고 분산전류원모 델인 LORETA-KEY (KEY institute for Brain-Mind Research, Switzerland) 소프트웨어를 사용하였다. 주파수는 7개의 영역으 로 나누었다: 델타(1 6 Hz), 테타(6 8 Hz), 알파-1 $(8 \sim 10 \mathrm{~Hz})$, 알파-2 (10 12 Hz), 베타-1 (12 18 Hz), 베타-2 $(18 \sim 21 \mathrm{~Hz})$, 그 리고 베타-3 $(21 \sim 30 \mathrm{~Hz})$. 본 연구에서 사용한 LORETA의 버전은 몬트리올신경과학연구소(Montreal Neurologic Institute)의 뇌 영상센터에서 디지털화된 자기공명영상으로 만들어진 Talairach 인간뇌지도(Talairach, 1988)에 등록된 세껍질둥근머리모델 (three-shell spherical head model)을 사용하였다. 세껍질둥근 머리모델과 Talairach 머리구조(Talairach head geometry) 사이 의 등록은 Towle (Towle VL와 DN, 1993)에 의해 발표된 실질적 뇌파전기 좌표를 사용하였다. LORETA 영상은 Talairach 도감의 뇌겉질과 해마에 국한되고, $7 \mathrm{~mm}$ 범위의 공간 해상도를 가지며 총 2,394개의 격자(voxel) 안에 표시된다(Pascual-Marqui, 1994; $\mathrm{Kim}, 2002)$. 테니스심상훈련을 하는 동안 기록된 뇌파에서 잘라낸 뇌파절편들과 휴식-II에서 기록한 뇌파에서 잘라낸 뇌파절편 사이 에서 통계비모수지도(statistical-nonparametric map, SnPMs) 을 구하였다. 통계적 유의성은 $p<0.01$ 로 하였다.

\section{결 과}

테니스 심상훈련에 의한, 오른쪽 대뇌반구의 해마, 해마옆이랑, 그리고 후두부의 방추모양이랑에서 알파-2 영역의 전류원밀도가 의미 있게 증가하였다 $(p<0.01)$ (Fig. 1, Table 1). 알파-2 영역을 

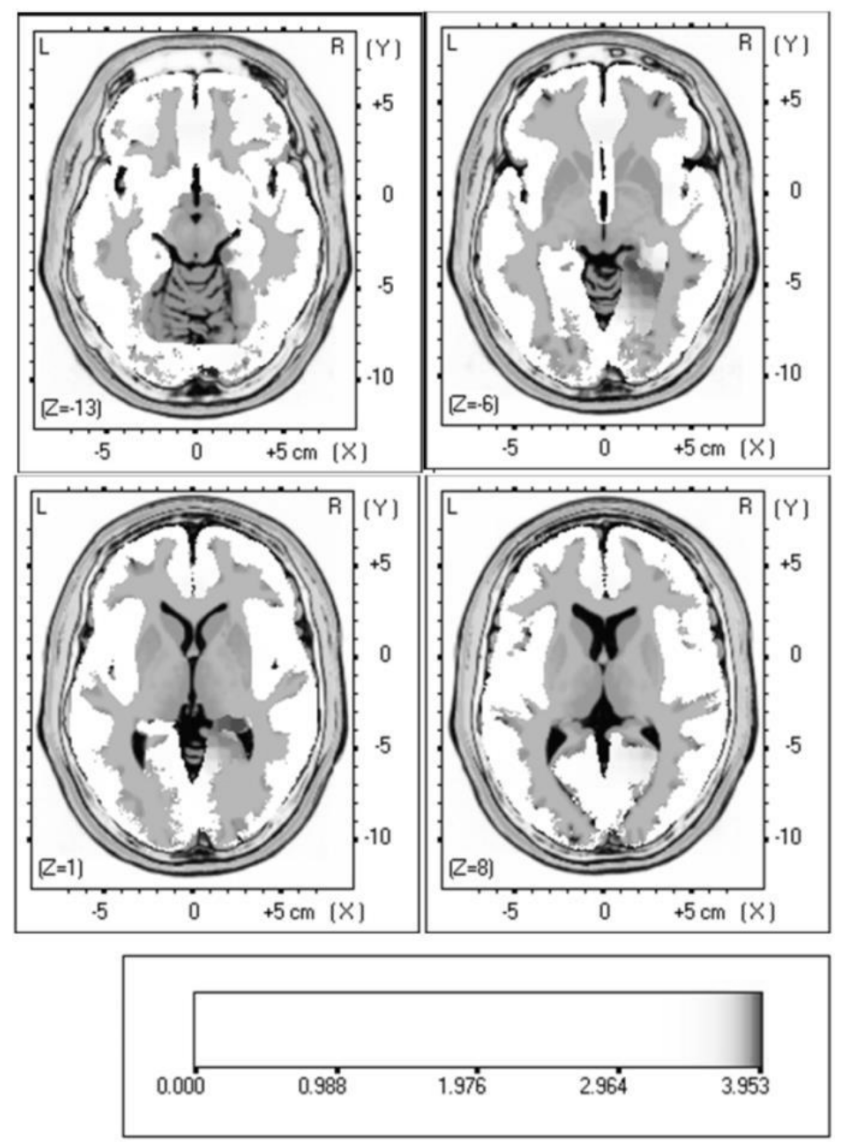

Fig. 1. Statistical-nonparametric maps of the alpha-2 frequency band between the imagery tennis playing and the resting in healthy and right-handed young males $(n=11)$. The current-source density was increased in the parahippocampal gyrus, hippocampus and fusiform gyrus of the right cerebral hemisphere by the imagery tennis playing $(p<0.01)$.

제외한 나머지 주파수영역에서는 유의한 변화가 관찰되지 않았다.

\section{고 찰}

저자들은 테니스심상훈련을 하면서 뇌파를 기록하고 이용하여 얻은 분산전류원모델을 이용하여 뇌파의 디지털데이타를 주파수 영역별로 분석하였다. 이 분석을 통해서 심상훈련이 뇌파 전류원에 어떠한 변화를 일으키는지 주파수영역별로 관찰하였다. 풍경사진 슬라이드 쇼를 보고나서 휴식하는 상태와 테니스비디오클립을 보 고나서 테니스심상훈련을 하는 상태의 뇌파절편을 분석하여 통계 비모수지도를 얻어서 각주파수별로 전류원밀도분포의 차이를 관 찰하였다. 의미 있는 차이를 보인 주파수영역은 알파-2 (10 12 $\mathrm{Hz}$ ) 였고, 오른쪽 대뇌반구의 해마, 해마옆이랑, 그리고 후두부의 방추모양이랑에서 전류원밀도가 증가되었다. 오른쪽 대뇌반구의 해마옆이랑은 장면을 인지하는 기능이 있고(Aguirre, 1996; Aguirre,
Table 1. Maximal-points of current-source density of alpha-2 frequency band on the SnPMs $(p<0.01)$ between the imagery-tennis playing and the resting state in healthy and right-handed young males $(n=11)$

\begin{tabular}{|c|c|c|}
\hline $\begin{array}{l}\text { Talairach coordinate } \\
\qquad(x, y, z)\end{array}$ & Anatomical location & t-value \\
\hline$X=18, Y=-39, Z=-6$ & $\begin{array}{l}\text { Parahippocampal } \\
\text { gyrus, right }\end{array}$ & $3.952500 \mathrm{E}+0000$ \\
\hline$X=25, Y=-39, Z=1$ & Hippocampus, right & $3.952500 E+0000$ \\
\hline$X=32, Y=-53, Z=1$ & $\begin{array}{l}\text { Parahippocampal } \\
\text { gyrus, right }\end{array}$ & $3.937000 E+0000$ \\
\hline$X=32, Y=-53, Z=-6$ & Fusiform gyrus, right & $3.937000 E+0000$ \\
\hline
\end{tabular}

SnPMs, statistical-nonparametric maps.

1998; Epstein, 1998; Ishai, 1999), 오른쪽 해마는 공간 검색의 기 능이 있으며(Maguire, 1997), 오른쪽 방추모양이랑은 사물을 인지 하는 기능이 있는 것으로(Behrmann, 1994) 이전 연구들에서 알려 져 있다. 본 연구의 결과는 장면인지, 공간검색, 사물인지 등의 심상 훈련에 필요한 기능이 있는 해마/해마옆이랑-후두엽 사이의 알파 신경망이 테니스심상훈련에 의해 활성화된다는 점을 시사하였다.

알파진동의 동기화는 인지과정 중에 깨지므로 알파리듬의 고전 적 개념은 휴식을 의미한다. 저주파알파리듬 $(7 \sim 10 \mathrm{~Hz})$ 의 비동기 화는 거의 모든 인지과정에서 나타나고 분포도 넓다. 주의집중 과 정이나 일반적 과제를 수행하고자 하는 의지에 의해서도 저주파알 파리듬의 비동기화는 발생한다. 고주파알파리듬 $(10 ~ 12 \mathrm{~Hz})$ 의 비 동기화는 감각, 어의 정보에 의해 발생 두정엽 - 후두엽에서 잘 나타 난다(Klimesch, 1993; Klimesch, 1994; Klimesch, 1996; Klimesch, 1997). 알파리듬의 비동기화는 뇌가 활성화된 상태를 의미하고 알파리듬의 동기화는 뇌겉질의 흥분성이 감소되거나 신 경세포 군집이 억제되는 상황이다(Pfurtscheller, 1999).

최근에는 알파리듬이 신경망의 활성화를 의미한다는 견해가 있 다. 감각자극에 의해 발생한 알파반응은 자극이 끝나고 200 300 $\mathrm{ms}$ 가 지나면 기저치로 돌아간다(Basar E, 1972). 사건관련-비동 기화가 발생하는 경우에도 곧 이어 사건관련-동기화가 뒤따라 나 타난다(Pfurtscheller G, 1999). 본 연구에서 알파-2 영역 전류원 의 증가는 사건관련-비동기화에 따라 나오는 사건관련-동기화의 반영으로 판단하였다. 고양이에게 후두엽, 시상, 그리고 청각겉질 에 전극을 넣고 시각자극에 의한 유발전위를 관찰하였더니 시각자 극에 대해 8 15 Hz의 알파진동이 증가되었다. 또한 해마-후두엽 과 시상-후두엽 사이에서 알파진동의 뇌파-동시성이 증가하였으 며 특히 해마-후두엽의 뇌파-동시성이 현저하게 증가되었다. 본 연 구에서도 해마-후두엽의 알파 신경망이 활성화 되어 일치된 결과 를 보였다(Schurmann 등, 2000).

표준 10-20체계 21채널에서 얻은 뇌파의 디지털데이터를 네 껍질둥근머리모델을 이용해 분석하여 쌍극자전류원을 분석하면 
평균 $17 \mathrm{~mm}$ 의 국소화오차(localization error)와 평균 $31^{\circ}$ 의 방 향오차(orientation error)가 발생한다고 보고되었다(Krings 등, 1999). 세껍질둥근머리모델을 사용하며 분산전류원모델인 LORETA 는 두피뇌파의 측정 시 전극수가 25 개에서 89 개까지 증가하면서 국소화의 정확도도 증가하지만 그 이상의 전극 수에서는 비슷한 정 확도를 나타낸다(Michel 등, 2004). 뇌파의 디지털데이터를 세껍 질둥근머리모델을 사용하여 LORETA로 분석하여 전류원을 국소 화할 때 전극을 머리 전체에 균일하게 분포시키면 19채널과 46채 널 사이에 비슷한 전류원밀도분포를 관찰할 수 있다(Michel 등, 2004). 본 연구에서는 비교적 적은 수인 25 채널의 뇌파 데이터를 분석하였고 실제머리모델을 사용하지 못한 한계를 가지고 있다. 그 러나 뇌전증병소의 전류원을 찾는 과정과는 달리, 인지기능을 연구 할 때에는 뇌의 엽(lobes) 수준의 해상 정보만으로도 유용한 정보를 얻을 수 있다는 사실과 위에 언급한 오차를 고려하면 결과를 해석 하는데 문제가 되지 않을 것으로 판단하였다.

\section{Acknowledgements: None \\ Funding: None \\ Conflict of interest: None}

\section{References}

1. Aguirre GK1, Detre JA, Alsop DC, D'Esposito M. The parahippocampus subserves topographical learning in man. Cereb Cortex 1996;6(6):823-829.

2. Aguirre GK1, Zarahn E, D'Esposito M. An area within human ventral cortex sensitive to "building" stimuli: evidence and implications. Neuron 1998;21(2):373-383.

3. Atienza FL, Balaguer I, Garcia-Merita ML. Video modeling and imaging training on performance of tennis service of 9- to 12-year-old children. Percept Mot Skills 1998;87(2):519-529.

4. Başar E, Ozesmi C. The hippocampal EEG-activity and a systems analytical interpretation of averaged evoked potentials of the brain. Kybernetik 1972;12(1):45-54.

5. Behrmann M1, Moscovitch M, Winocur G. Intact visual imagery and impaired visual perception in a patient with visual agnosia. J Exp Psychol Hum Percept Perform 1994;20(5):1068-1087.

6. Chapman LJ, Chapman JP. The measurement of handedness. Brain Cogn 1987;6(2):175-183.

7. Cho HY, Kim JS, and LEE G C. Effects of motor imagery training on balance and gait abilities in post-stroke patients: a randomized controlled trial. Clin Rehabil 2013; 27:675-680

8. Farah MJ, Peronnet F, Gonon MA, Giard MH. Electrophysiological evidence for a shared representational medium for visual images and visual percepts. J Exp Psychol Gen 1988;117(3):248-257.

9. Farah MJ, Soso MJ, Dasheiff RM. Visual angle of the mind's eye before and after unilateral occipital lobectomy. J Exp Psychol Hum Percept Perform 1992;18(1):241-246.
10. Goldenberg G, Podreka I, Steiner M, Willmes K, Suess E, Deecke L. Regional cerebral blood flow patterns in visual imagery. Neuropsychologia 1989;27(5):641-664.

11. Ishai $A^{1}$, Ungerleider LG, Martin A, Schouten JL, Haxby JV. Distributed representation of objects in the human ventral visual pathway. Proc Natl Acad Sci US A 1999;96(16):9379-9384.

12. Jackson PL, Lafleur MF, Malouin F, Richards C, Doyon J. Potential role of mental practice using motor imagery in neurologic rehabilitation. Arch Phys Med Rehabil 2001;82(8):1133-1141.

13. Klimesch $W^{1}$, Doppelmayr M, Pachinger T, Ripper B. Brain oscillations and human memory: EEG correlates in the upper alpha and theta band. Neurosci Lett 1997;238(1-2):9-12.

14. Klimesch W, Schimke H, Doppelmayr M, Ripper B, Schwaiger J, Pfurtscheller G, et al. Event-related desynchronization (ERD) and the Dm effect: does alpha desynchronization during encoding predict later recall performance?. Int J Psychophysiol 1996;24(1-2):47-60.

15. Klimesch W, Schimke H, Schwaiger J. Episodic and semantic memory: an analysis in the EEG theta and alpha band. Electroencephalogr Clin Neurophysiol 1994;91(6):428-441.

16. Kosslyn SM AN, Thompson WL, Malikovic V, Weise SB, Chabris $\mathrm{CF}$, et al. Visual mental imagery activates topographically organized visual cortex: PET investigations. J. Cogn. Neurosci 1993;5:263-287.

17. Krings T, Chiappa KH, Cuffin BN, Cochius JI, Connolly S, Cosgrove GR. Accuracy of EEG dipole source localization using implanted sources in the human brain. Clin Neurophysiol 1999;110(1):106-114.

18. Maguire EA, Frackowiak RS, Frith CD. Recalling routes around london: activation of the right hippocampus in taxi drivers. $J$ Neurosci 1997;17(18):7103-7110.

19. Michel CM, Murray MM, Lantz G, Gonzalez S, Spinelli L, Grave de Peralta R. EEG source imaging. Clin Neurophysiol 2004; 115(10):2195-2222.

20. MR Kim, KR Kim, CK Ha, SH Choi, IK Lee. Comparative study between visual analysis and low resolution electromagnetic tomography (LORETA) method in the localizaion of epileptiform discharges. J Korean Neurol Assoc 2002;20:164-168

21. Pascual-Marqui RD MC, Lehmann D. Low resolution electromagnetic tomography: a new method for localizing electrical activity in the brain. Int J Psychophysiol 1994;18::49-65.

22. Pascual-Marqui RD, Michel CM, Lehmann D. Low resolution electromagnetic tomography: a new method for localizing electrical activity in the brain. Int J Psychophysiol 1994;18:49-65.

23. Pfurtscheller G1, Lopes da Silva FH. Event-related EEG/MEG synchronization and desynchronization: basic principles. Clin Neurophysiol 1999;110(11):1842-1857.

24. R Epstein, N Kanwisher. A cortical representation of the local visual environment. Nature 1998;392(6676):598-601.

25. Schurmann M, Demiralp T, Basar E, Basar Eroglu C. Electroencephalogram alpha $(8-15 \mathrm{~Hz})$ responses to visual stimuli in cat cortex, thalamus, and hippocampus: a distributed alpha network? Neurosci Lett 2000;292(3):175-178.

26. Talairach J TP. Co-planar stereotaxic atlas of the human brain. 1988, Thieme, New York.

27. Tokumaru O MC, Takada Y, Ashida H. EEG activity of aviators during imagery flight training. Clin Neurophysiol 2003;114(10): 
1926-1935.

28. Towle VL, Bolanos J, Suarez D, Tan K, Grzeszczuk R, Levin DN, et al. The spatial location of EEG electrodes: locating the best-fitting sphere relative to cortical anatomy. Electroencephalogr Clin
Neurophysiol 1993;86:1-6.

29. W Klimesch, H Schimke, G Pfurtscheller, cognitive load and memory performance. Brain Topogr 1993;5(3):241-251. 